\title{
Ligand Influence on the Electronic Spectra of Dicationic Ruthenium- Bipyridine-Terpyridine Complexes
}

\author{
Shuang $X u^{1}$, James E. T. Smith ${ }^{2}$ and J. Mathias Weber ${ }^{2}$ \\ ${ }^{1}$ JILA and Department of Physics, University of Colorado, Boulder, CO 80309-0440, USA \\ ${ }^{2}$ JILA and Department of Chemistry and Biochemistry, University of Colorado, \\ Boulder, CO 80309-0440, USA
}

\section{Table of Contents}

Figure S1. Laser intensity dependence of fragment yields

Table S1. Lowest energy calculated electronic transition energies and oscillator strengths for $\mathrm{L}=\mathrm{H}_{2} \mathrm{O}$

Figure S3. Calculated orbitals contributing to transitions for $\mathrm{L}=\mathrm{H}_{2} \mathrm{O}$.

Table S2. Cartesian coordinates of $\left[\mathbf{R u}-\mathrm{OH}_{2}\right]^{2+}$ (in Bohr radii).

Table S3. Lowest energy calculated electronic transition energies and oscillator strengths for $\mathrm{L}=\mathrm{CH}_{3} \mathrm{CN}$

Figure S4. Calculated orbitals contributing to transitions for $\mathrm{L}=\mathrm{H}_{2} \mathrm{O}$.

Table S4. Cartesian coordinates of $\left[\mathbf{R u}-\mathrm{NCCH}_{3}\right]^{2+}$ (in Bohr radii).

Table S5. Lowest energy calculated electronic transition energies and oscillator strengths for $\mathrm{L}=\mathrm{CO}_{2}$

Figure S5. Calculated orbitals contributing to transitions for $\mathrm{L}=\mathrm{H}_{2} \mathrm{O}$.

Table S6. Cartesian coordinates of $[\mathbf{R u}-\mathrm{OCO}]^{2+}$ (in Bohr radii).

Table S7. Lowest energy calculated electronic transition energies and oscillator strengths for $\mathrm{L}=\mathrm{CO}_{2}$

Figure S6. Calculated orbitals contributing to transitions for $\mathrm{L}=\mathrm{H}_{2} \mathrm{O}$.

Table S8. Cartesian coordinates of $\left[\mathbf{R u}-\mathrm{NCCH}_{3}\right]^{2+}$ (in Bohr radii).

Table S9. Lowest energy calculated electronic transition energies and oscillator strengths for $\mathrm{L}=\mathrm{CH}_{3} \mathrm{CN}$

Figure S6. Calculated orbitals contributing to transitions for $\mathrm{L}=\mathrm{H}_{2} \mathrm{O}$. 
Figure S1. Light intensity dependence of fragment ion yield. The laser was set to the wavelength of peak absorption for each species. The full lines show linear fits, their length indicates which data points were included in the fitting.

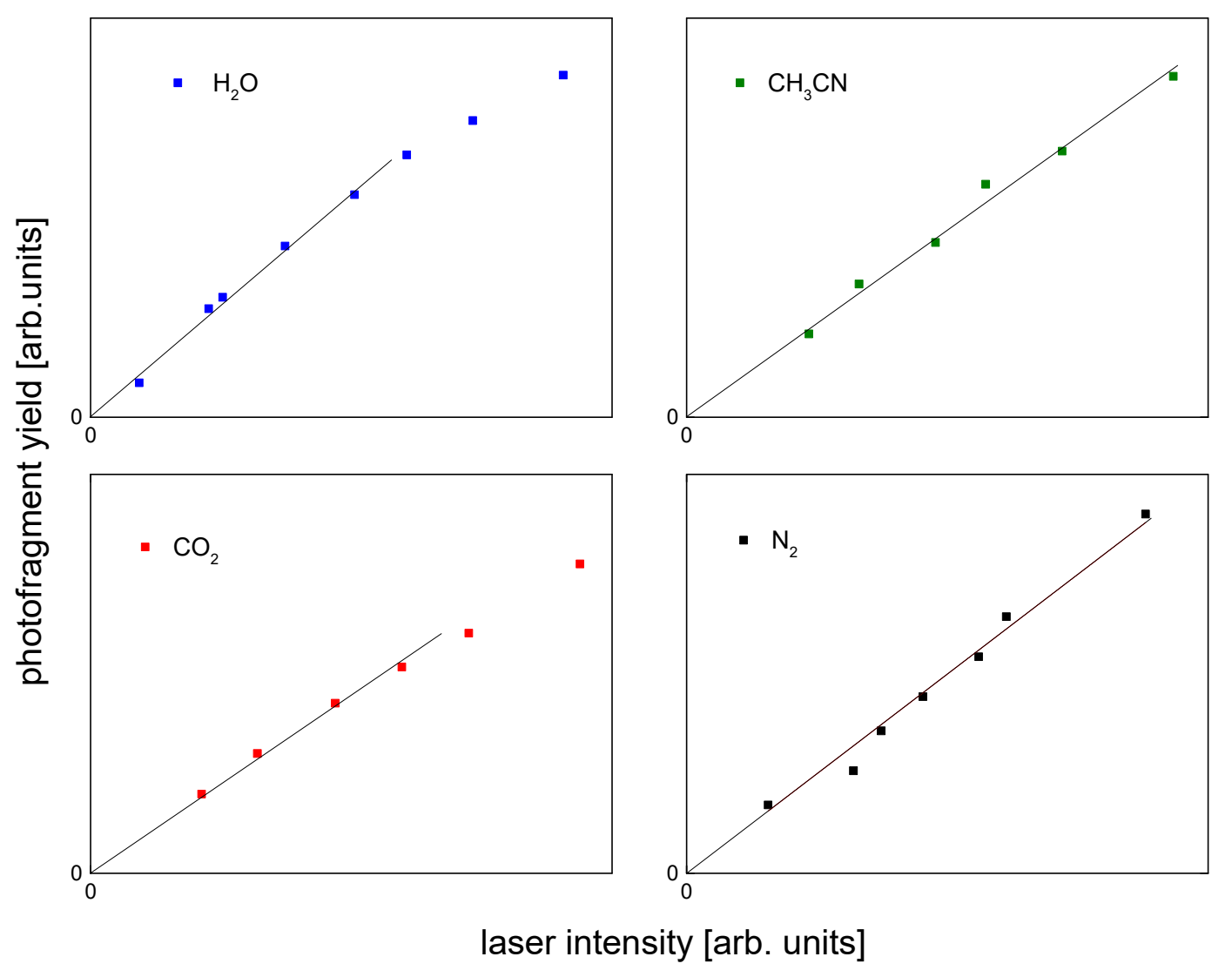


Figure S2. Calculated molecular orbital levels for $[\mathbf{R u}-\mathrm{L}]^{2+}$ and bare $\mathbf{R} \mathbf{u}^{2+}$.

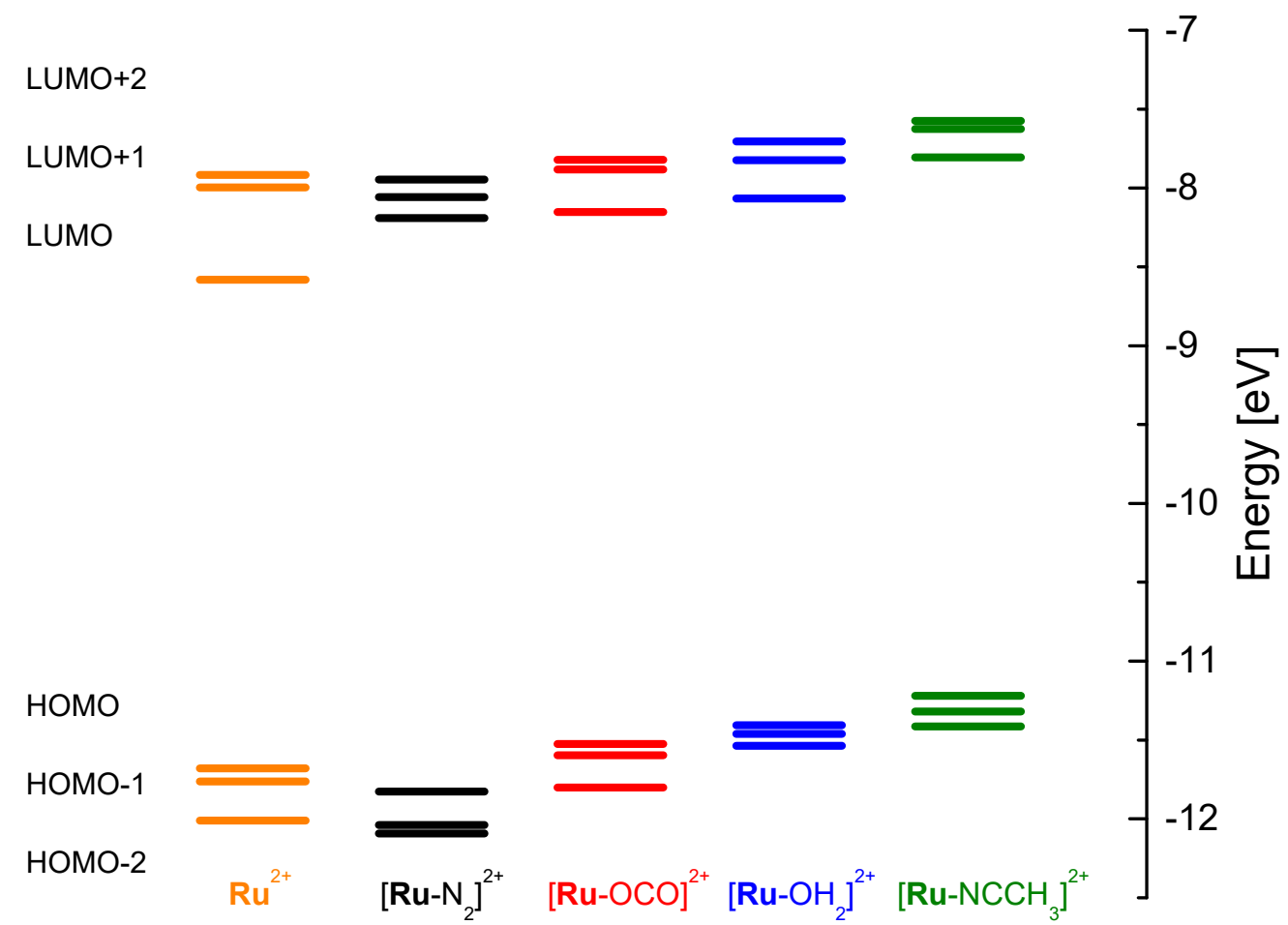


Table S1. Lowest energy calculated electronic transition energies and oscillator strengths for $\mathrm{L}=\mathrm{H}_{2} \mathrm{O}$ (B3LYP functional, unshifted). Only contributions of $10 \%$ and greater are shown.

\begin{tabular}{|c|c|c|c|c|}
\hline Transition & $\mathrm{E}[\mathrm{eV}] / \mathrm{f}\left[10^{-3}\right]$ & $\begin{array}{c}\mathrm{E}_{\exp }[\mathrm{eV}] \\
\pm 0.03 \mathrm{eV}\end{array}$ & $\begin{array}{c}\text { orbitals } \\
\text { (see p. S4) }\end{array}$ & character \\
\hline 1 & $2.33 / 5.26$ & 2.16 & $114 \rightarrow 115(96.2 \%)$ & $\mathrm{d} \rightarrow \operatorname{trpy}$ \\
\hline 2 & $2.45 / 8.50$ & 2.40 & $113 \rightarrow 115(95.9 \%)$ & $\mathrm{d} \rightarrow \operatorname{trpy}$ \\
\hline 3 & $2.69 / 11.5$ & 2.65 & $\begin{array}{l}112 \rightarrow 115(66.1 \%) \\
114 \rightarrow 116(24.5 \%)\end{array}$ & $\mathrm{d} \rightarrow \operatorname{trpy}$ \\
\hline 4 & $2.80 / 3.14$ & & $114 \rightarrow 117(86.8 \%)$ & $\mathrm{d} \rightarrow$ bipy \\
\hline 5 & $2.82 / 2.51$ & & $\begin{array}{l}114 \rightarrow 116(53.1 \%) \\
113 \rightarrow 116(26.2 \%)\end{array}$ & $\mathrm{d} \rightarrow \operatorname{trpy}$ \\
\hline 6 & $2.92 / 0.049$ & & $112 \rightarrow 117(91.3 \%)$ & $\mathrm{d} \rightarrow$ bipy \\
\hline 7 & $2.93 / 23.7$ & & $112 \rightarrow 116(92.4 \%)$ & $\mathrm{d} \rightarrow$ trpy \\
\hline 8 & $2.96 / 109$ & 2.81 & $\begin{array}{l}113 \rightarrow 116(56.8 \%) \\
113 \rightarrow 117(13.1 \%) \\
114 \rightarrow 116(12.7 \%) \\
112 \rightarrow 115(11.9 \%)\end{array}$ & $\mathrm{d} \rightarrow \operatorname{trpy}$ \\
\hline 9 & $2.13 / 16.1$ & 3.00 & $113 \rightarrow 117(69.7 \%)$ & $\mathrm{d} \rightarrow$ bipy \\
\hline 10 & $3.25 / 0.091$ & & $\begin{array}{l}113 \rightarrow 122(40.8 \%) \\
114 \rightarrow 122(27.0 \%) \\
113 \rightarrow 124(12.4 \%)\end{array}$ & $d \rightarrow$ water \\
\hline 11 & $3.47 / 1.34$ & & $\begin{array}{l}112 \rightarrow 122(64.8 \%) \\
112 \rightarrow 126(14.2 \%)\end{array}$ & $\mathrm{d} \rightarrow$ water \\
\hline
\end{tabular}


Figure S2. Calculated orbitals contributing to transitions for $\mathrm{L}=\mathrm{H}_{2} \mathrm{O}$. Orbital numbers are given as mentioned in Table S1.
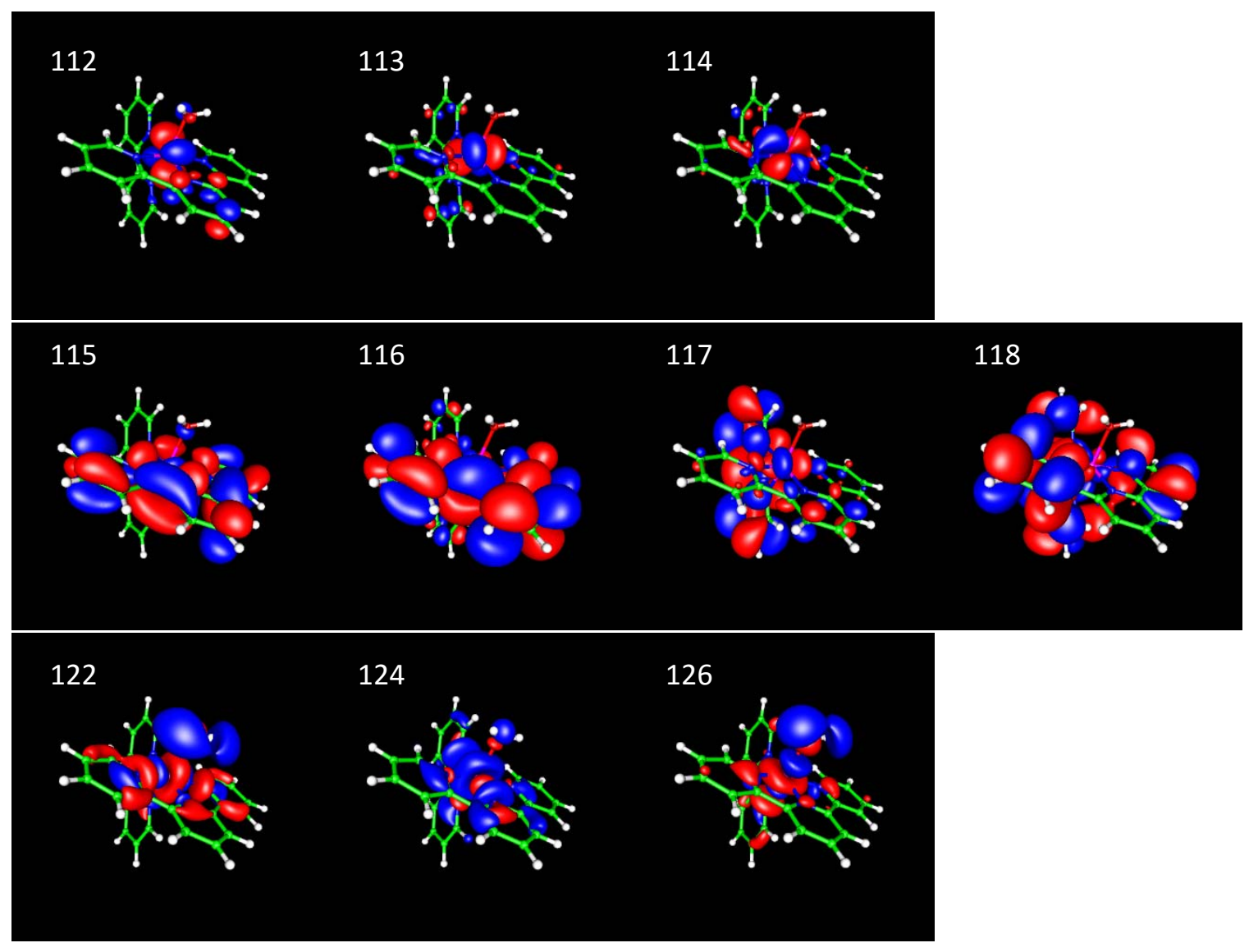
Table S2. Cartesian coordinates of $\left[\mathbf{R u}-\mathrm{OH}_{2}\right]^{2+}$ (in Bohr radii).

$-0.64398958121267-3.92800332423659 \quad-0.82597180993828 \mathrm{Ru}$

$\begin{array}{lll}-1.04620306455772 & -2.37543072241385 & 7.31238331623689 \mathrm{C}\end{array}$

$\begin{array}{lll}-3.47012810779927 & -1.40007064795537 & 7.57136720374500 \mathrm{C}\end{array}$

$-4.97825738801101-1.18451001588155 \quad 5.44073307912922 \mathrm{C}$

$-4.04527935673381-1.94280699161948 \quad 3.09865853087940$ C

$-0.22363776337256 \quad-3.09659630422191 \quad 4.93657437755193$ C

$0.19810911322174 \quad-2.57928620806929 \quad 8.92038571260517 \mathrm{H}$

$-4.17538082207136-0.81750650270942 \quad 9.39980440608995 \mathrm{H}$

$-6.86711311490438-0.43049674516757 \quad 5.60830026463695 \mathrm{H}$

$\begin{array}{lll}1.64697030912348 & -3.86505575356537 & 4.64760758307132 \mathrm{H}\end{array}$

$\begin{array}{lll}-1.67448431811001 & -2.89308047075067 & 2.86402090959486 \mathrm{~N}\end{array}$

$\begin{array}{lll}-7.93987642994805 & -0.87760985298871 & 0.55912958806792 \\ \mathrm{C}\end{array}$

$-9.16163746515616-0.80472754547642 \quad-1.75446246529200 \mathrm{C}$

$-7.88448642786204-1.66283438500318-3.87871853598618 \mathrm{C}$

$-5.44406980992593 \quad-2.55750058809518 \quad-3.60741685158354$ C

$\begin{array}{lll}-5.48070222449675 & -1.79792795511328 & 0.72593443386664 \mathrm{C}\end{array}$

$\begin{array}{lll}-8.89978050103913 & -0.21830783255590 & 2.23460349862715 \mathrm{H}\end{array}$

$-11.07242896418107-0.09158145306444 \quad-1.89417065946040 \mathrm{H}$

$-8.75296041130058-1.64588688303114 \quad-5.72815265537685 \mathrm{H}$

$-4.39811587382806-3.23839681478604-5.22157807660347 \mathrm{H}$

$-4.24391051692626-2.63533000510117 \quad-1.37061947423980 \mathrm{~N}$

$\begin{array}{lll}3.18046293151725 & -5.18267135323163 & 0.38545526340684 \mathrm{O}\end{array}$

$3.62025063199517 \quad-6.95243748206088 \quad 0.28172999251335 \mathrm{H}$

$4.64669084381057-4.23949371101090 \quad-0.15949088985916 \quad \mathrm{H}$

$\begin{array}{lll}-3.62418701162497 & -11.58246081029552 & 0.67213956176024 \mathrm{C}\end{array}$

$\begin{array}{llll}-3.26653774192277 & -12.73660722504203 & -1.65353397530480 \mathrm{C}\end{array}$

$-2.17541460740676-11.35473074176052-3.59829429395088$ C

$\begin{array}{lll}-1.46667384597441 & -8.85908355305670 & -3.19148910799030 \mathrm{C}\end{array}$

$\begin{array}{lll}-2.88307360838078 & -9.08709811184569 & 0.96992399627046 \mathrm{C}\end{array}$ 


\begin{tabular}{lllll}
-4.46541785535192 & -12.58343565495499 & $2.24244318180189 \mathrm{H}$ \\
-3.82591643570782 & -14.67980600869813 & $-1.95631336114009 \mathrm{H}$ \\
-1.88385214593024 & -12.22039865696021 & $-5.42463390836100 \mathrm{H}$ \\
-3.13359723974991 & -8.12144001152238 & $2.75309471163025 \mathrm{H}$ \\
-1.82974567928211 & -7.73282236742337 & $-0.89331994861512 \mathrm{~N}$ \\
0.35443883562323 & -7.97712404002784 & $-7.59455057014416 \mathrm{C}$ \\
1.48687503219930 & -6.21965211294723 & $-9.18522793765483 \mathrm{C}$ \\
1.97725159106072 & -3.78460817265200 & $-8.32963722094733 \mathrm{C}$ \\
1.30669456433752 & -3.13897844933957 & $-5.86960541580289 \mathrm{C}$ \\
-0.28738202233960 & -7.25531285556626 & $-5.14805729830332 \mathrm{C}$ \\
-0.00575593418114 & -9.87324980260202 & $-8.25960191243971 \mathrm{H}$ \\
1.99797626027531 & -6.75223125466116 & $-11.09157806985102 \mathrm{H}$ \\
\hline 2.87404591278927 & -2.42921912299223 & $-9.56500627898829 \mathrm{H}$ \\
0.18694964842691 & -4.87230551301590 & $-4.36889788945295 \mathrm{~N}$ \\
\hline 2.76962403522833 & 1.40309281886488 & $-5.83579805747768 \mathrm{C}$ \\
3.08105188334053 & 3.65772393566940 & $-4.53098343548816 \mathrm{C}$ \\
\hline 2.31309764724816 & 3.78724631467208 & $-2.02840960338984 \mathrm{C}$ \\
1.25953439329302 & 1.65937869971139 & $-0.91991738158385 \mathrm{C}$ \\
\hline 1.70214841822605 & -0.67029541919097 & $-4.62827619973772 \mathrm{C}$ \\
3.35426556708171 & 1.26623420391717 & $-7.78731225752643 \mathrm{H}$ \\
3.90855859255336 & 5.28101758795938 & $-5.45875525786005 \mathrm{H}$ \\
2.51773774364404 & 5.50211397709305 & $-0.93615302071501 \mathrm{H}$ \\
0.63683004572475 & 1.68637931250069 & $1.02599120364281 \mathrm{H}$ \\
\hline .94862426856866 & -0.52762541772367 & $-2.15945399406305 \mathrm{~N}$
\end{tabular}


Table S3. Lowest energy calculated electronic transition energies and oscillator strengths for $\mathrm{L}=\mathrm{CH}_{3} \mathrm{CN}$ (B3LYP functional, unshifted). Only contributions of $10 \%$ and greater are shown.

\begin{tabular}{|c|c|c|c|c|}
\hline Transition & $\mathrm{E}[\mathrm{eV}] / \mathrm{f}\left[10^{-3}\right]$ & $\begin{array}{c}E_{\text {exp }}[\mathrm{eV}] \\
\pm 0.03 \mathrm{eV} \\
\end{array}$ & $\begin{array}{c}\text { orbitals } \\
\text { (see p. S8) }\end{array}$ & character \\
\hline 1 & $2.47 / 5.14$ & & $120 \rightarrow 121(90.8 \%)$ & $\mathrm{d} \rightarrow$ trpy \\
\hline 2 & $2.58 / 5.89$ & & $119 \rightarrow 121(90.8 \%)$ & $\mathrm{d} \rightarrow \operatorname{trpy}$ \\
\hline 3 & $2.77 / 1.04$ & & $\begin{array}{l}120 \rightarrow 123(76.1 \%) \\
120 \rightarrow 122(21.6 \%)\end{array}$ & $\mathrm{d} \rightarrow \operatorname{trpy}$ \\
\hline 4 & $2.80 / 2.78$ & & $\begin{array}{l}120 \rightarrow 122(48.0 \%) \\
118 \rightarrow 121(38.6 \%) \\
120 \rightarrow 123(12.0 \%)\end{array}$ & $\mathrm{d} \rightarrow$ bipy \\
\hline 5 & $2.89 / 13.8$ & & $\begin{array}{l}119 \rightarrow 122(33.7 \%) \\
119 \rightarrow 123(27.0 \%) \\
118 \rightarrow 121(23.4 \%) \\
120 \rightarrow 122(12.6 \%)\end{array}$ & $\mathrm{d} \rightarrow$ trpy \\
\hline 6 & $2.96 / 0.12$ & & $\begin{array}{l}118 \rightarrow 123(71.2 \%) \\
118 \rightarrow 122(26.9 \%)\end{array}$ & $d \rightarrow$ bipy \\
\hline 7 & $2.98 / 108$ & & $\begin{array}{l}119 \rightarrow 122(64.3 \%) \\
118 \rightarrow 121(12.6 \%) \\
120 \rightarrow 122(10.1 \%)\end{array}$ & $\mathrm{d} \rightarrow$ trpy \\
\hline 8 & $3.02 / 2.12$ & & $\begin{array}{l}118 \rightarrow 122(71.3 \%) \\
118 \rightarrow 123(26.2 \%)\end{array}$ & $\mathrm{d} \rightarrow$ trpy \\
\hline 9 & $3.16 / 2.17$ & & $\begin{array}{l}119 \rightarrow 123(57.6 \%) \\
118 \rightarrow 121(20.0 \%)\end{array}$ & $d \rightarrow$ bipy \\
\hline
\end{tabular}


Figure S3. Calculated orbitals contributing to transitions for $\mathrm{L}=\mathrm{CH}_{3} \mathrm{CN}$. Orbital numbers are given as mentioned in Table S3.

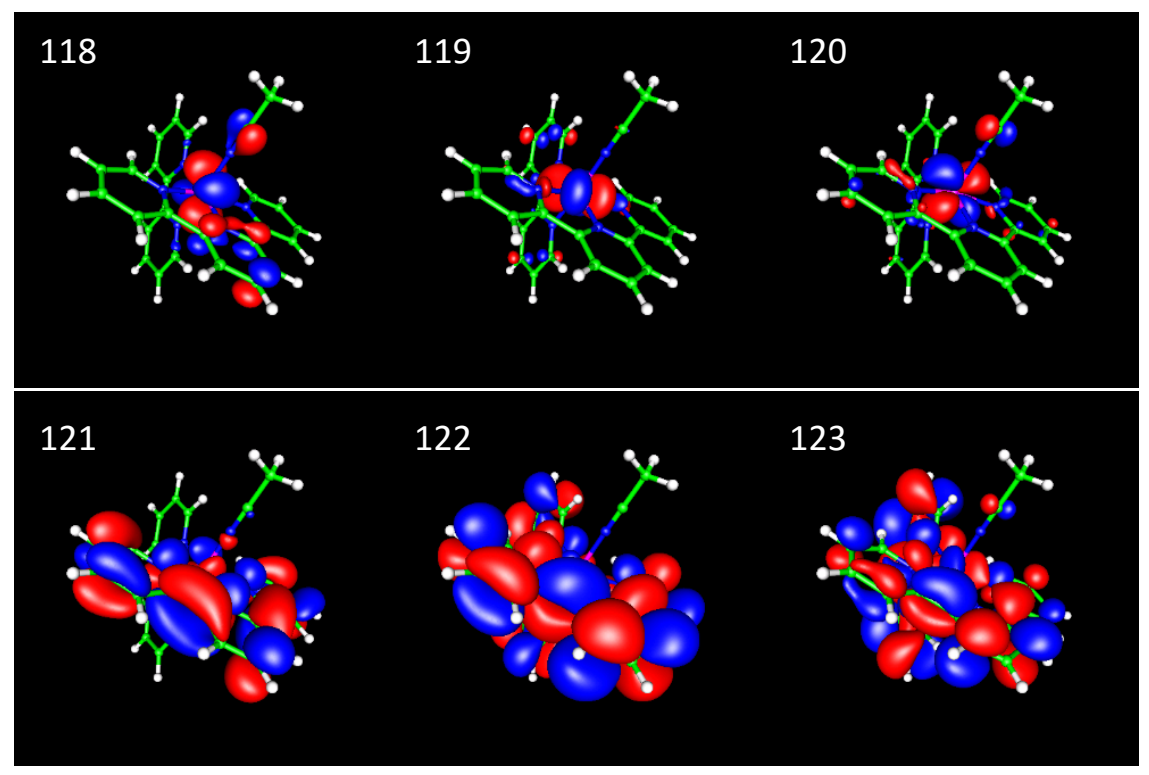


Table S4. Cartesian coordinates of $\left[\mathbf{R u}-\mathrm{NCCH}_{3}\right]^{2+}$ (in Bohr radii).

\begin{tabular}{|c|c|c|}
\hline-0.77875884891424 & -4.13185849973641 & $-0.85616583667320 \mathrm{R}$ \\
\hline-1.37433057244652 & -2.54022592893425 & $7.28385087611001 \mathrm{C}$ \\
\hline-3.78819022355918 & -1.52901599383063 & $7.48638995119035 \mathrm{C}$ \\
\hline-5.23993130987151 & -1.29911077111521 & 5.31971661167632 \\
\hline-4.26671136616785 & -2.07663944454292 & $2.99949461230996 \mathrm{C}$ \\
\hline-0.51133238254413 & -3.27877827624293 & 4.92861461576991 \\
\hline-0.16911495719109 & -2.75883809159660 & $4361 \mathrm{H}$ \\
\hline-4.52762145936147 & -0.93081031301468 & $9.29624105915017 \mathrm{H}$ \\
\hline-7.12054886864389 & -0.51716185828691 & $5.44102914608357 \mathrm{H}$ \\
\hline 1.35329818454755 & -4.07454788223036 & $4.69282010827897 \mathrm{H}$ \\
\hline-1.90415635681051 & -3.06299549626763 & $2.81746661199834 \mathrm{~N}$ \\
\hline-8.10971111047817 & -0.94562458179266 & $0.40916756929595 \mathrm{C}$ \\
\hline-9.29557505528294 & -0.84778605853015 & $3629625765 \mathrm{C}$ \\
\hline-8.00181695898127 & -1.71796640758949 & $3708065607 \mathrm{C}$ \\
\hline-5.57933445420718 & -2.65027215692894 & -3.72331705611934 \\
\hline-5.66611376697000 & -1.90480261631081 & 0.60405079190861 \\
\hline-9.08732954938986 & -0.27609008232316 & $2.06999158088084 \mathrm{H}$ \\
\hline-11.19346829705428 & -0.10566049693804 & -2.08698678652330 \\
\hline-8.84334226963089 & -1.68153013462544 & -5.89 \\
\hline-4.51981016642803 & -3.34246090150357 & $-5.32466079670701 \mathrm{H}$ \\
\hline-4.41493381341680 & -2.75351668684556 & $-1.47186057667826 \mathrm{~N}$ \\
\hline-3.95564959647897 & -11.72204986248412 & 0.56366001852117 \\
\hline-3.61597287671748 & -12.86414494439452 & -1.77004560349956 \\
\hline-2.49922322064741 & -11.48658001189767 & -3.70255657074146 \\
\hline-1.74845225461068 & -9.00672580486321 & $-3.27451962347671 \mathrm{C}$ \\
\hline-3.16622209142953 & -9.24340834345037 & 0.88129662663778 \\
\hline-4.81614016290694 & -12.71973472546649 & 2.12551759623611 \\
\hline-4.20822240174722 & -14.79506050205815 & -2.08824313805426 \\
\hline-2.21921945663561 & -12.34332997190026 & -5.53483988625017 \\
\hline
\end{tabular}




\begin{tabular}{|c|c|c|}
\hline-3.39265309056291 & -8.28928165145811 & $2.67387948821148 \mathrm{H}$ \\
\hline & -7.89755730974241 & - -0.97006649165263 \\
\hline 0.02629687057056 & -8.10273620021102 & -7.69250399876909 \\
\hline 1.16801053557153 & -6.34532282507863 & -9.27606634807649 \\
\hline & -3.93372893456219 & -8.39546633314331 \\
\hline 1.11729562203196 & -3.31378702369140 & -5.91220309834066 \\
\hline 55037727510499 & -7.40630339200029 & -5.2 \\
\hline-0.39540164168003 & -9.97729444828583 & $-8.38159117697986 \mathrm{H}$ \\
\hline 1.629 & -6.8 & \\
\hline 2.61815894601741 & -2.57565205506112 & \\
\hline 276 & -5.051 & -4. \\
\hline & & \\
\hline & 3.4 & -4.51880 \\
\hline & & \\
\hline 344 & 1.433 & -0.9 \\
\hline & -0.86 & -4.6 \\
\hline 3.2495874 & 1.063 & -7.7960 \\
\hline 3.88886901 & & $858 \mathrm{l}$ \\
\hline 2.51810466760666 & 5.25305293230499 & -0.90551 \\
\hline & & \\
\hline & -0.72977883789749 & -2.17890431 \\
\hline 27879048276 & -5.389 & \\
\hline 7.23052450159072 & -7.02417907001116 & 1.27633052926624 \\
\hline 8.0627 & -5.8331 & 2.74000766915726 \\
\hline 44530821353558 & -6.97693606404397 & -0.3892 \\
\hline 7.12276796196885 & -8.96278855756048 & 1.97088134741930 \\
\hline 4.72370602387238 & -6.11418528752113 & 0.6345115183613 \\
\hline
\end{tabular}


Table S5. Lowest energy calculated electronic transition energies and oscillator strengths for $\mathrm{L}=\mathrm{CO}_{2}$ (B3LYP functional, unshifted). Only contributions of $10 \%$ and greater are shown.

\begin{tabular}{|c|c|c|c|c|}
\hline Transition & $\begin{array}{c}\mathrm{E}[\mathrm{eV}] / \mathrm{f}\left[10^{-3}\right] \\
\mathrm{B} 3 \mathrm{LYP}\end{array}$ & $\begin{array}{l}E_{\exp }[\mathrm{eV}] \\
\pm 0.03 \mathrm{eV} \\
\end{array}$ & $\begin{array}{c}\text { orbitals } \\
\text { (see p. S4) } \\
\end{array}$ & character \\
\hline 1 & $2.34 / 7.75$ & & $120 \rightarrow 121(92.9 \%)$ & $\mathrm{d} \rightarrow \operatorname{trpy}$ \\
\hline 2 & $2.46 / 5.28$ & & $119 \rightarrow 121(91.6 \%)$ & $d \rightarrow$ trpy \\
\hline 3 & $2.78 / 10.5$ & & $\begin{array}{l}118 \rightarrow 121(52.9 \%) \\
120 \rightarrow 122(34.1 \%)\end{array}$ & $d \rightarrow$ trpy \\
\hline 4 & $2.79 / 0.22$ & & $\begin{array}{l}120 \rightarrow 123(78.2 \%) \\
120 \rightarrow 122(15.1 \%)\end{array}$ & $\mathrm{d} \rightarrow$ bipy \\
\hline 5 & $2.91 / 7.2$ & & $\begin{array}{l}120 \rightarrow 122(34.9 \%) \\
119 \rightarrow 122(23.3 \%) \\
119 \rightarrow 123(16.8 \%) \\
120 \rightarrow 123(11.5 \%) \\
118 \rightarrow 121(11.0 \%)\end{array}$ & $d \rightarrow$ trpy \\
\hline 6 & $2.99 / 0.26$ & & $\begin{array}{l}118 \rightarrow 123(29.2 \%) \\
120 \rightarrow 128(27.9 \%) \\
119 \rightarrow 128(15.0 \%) \\
118 \rightarrow 122(10.7 \%)\end{array}$ & $\mathrm{d} \rightarrow$ bipy \\
\hline 7 & $3.01 / 110$ & & $119 \rightarrow 122(73.9 \%)$ & $\mathrm{d} \rightarrow$ trpy \\
\hline 8 & $3.07 / 0.16$ & & $\begin{array}{l}118 \rightarrow 123(37.2 \%) \\
119 \rightarrow 128(26.2 \%) \\
118 \rightarrow 122(15.7 \%)\end{array}$ & $d \rightarrow$ trpy \\
\hline 9 & $3.12 / 11.7$ & & $\begin{array}{l}119 \rightarrow 123(59.4 \%) \\
118 \rightarrow 128(20.0 \%)\end{array}$ & $\mathrm{d} \rightarrow$ bipy \\
\hline 10 & $3.13 / 16.8$ & & $\begin{array}{l}118 \rightarrow 122(70.7 \%) \\
118 \rightarrow 123(21.1 \%)\end{array}$ & $\mathrm{d} \rightarrow$ trpy \\
\hline 11 & $3.32 / 0.72$ & & $\begin{array}{l}120 \rightarrow 129(36.8 \%) \\
119 \rightarrow 128(33.3 \%)\end{array}$ & \\
\hline 12 & $3.36 / 5.48$ & & $\begin{array}{l}118 \rightarrow 128(50.3 \%) \\
118 \rightarrow 121(17.6 \%) \\
119 \rightarrow 125(13.3 \%)\end{array}$ & $\mathrm{d} \rightarrow \mathrm{CO}_{2}$ \\
\hline
\end{tabular}


Figure S4. Calculated orbitals contributing to transitions for $\mathrm{L}=\mathrm{CO}_{2}$. Orbital numbers are given as mentioned in Table S5.

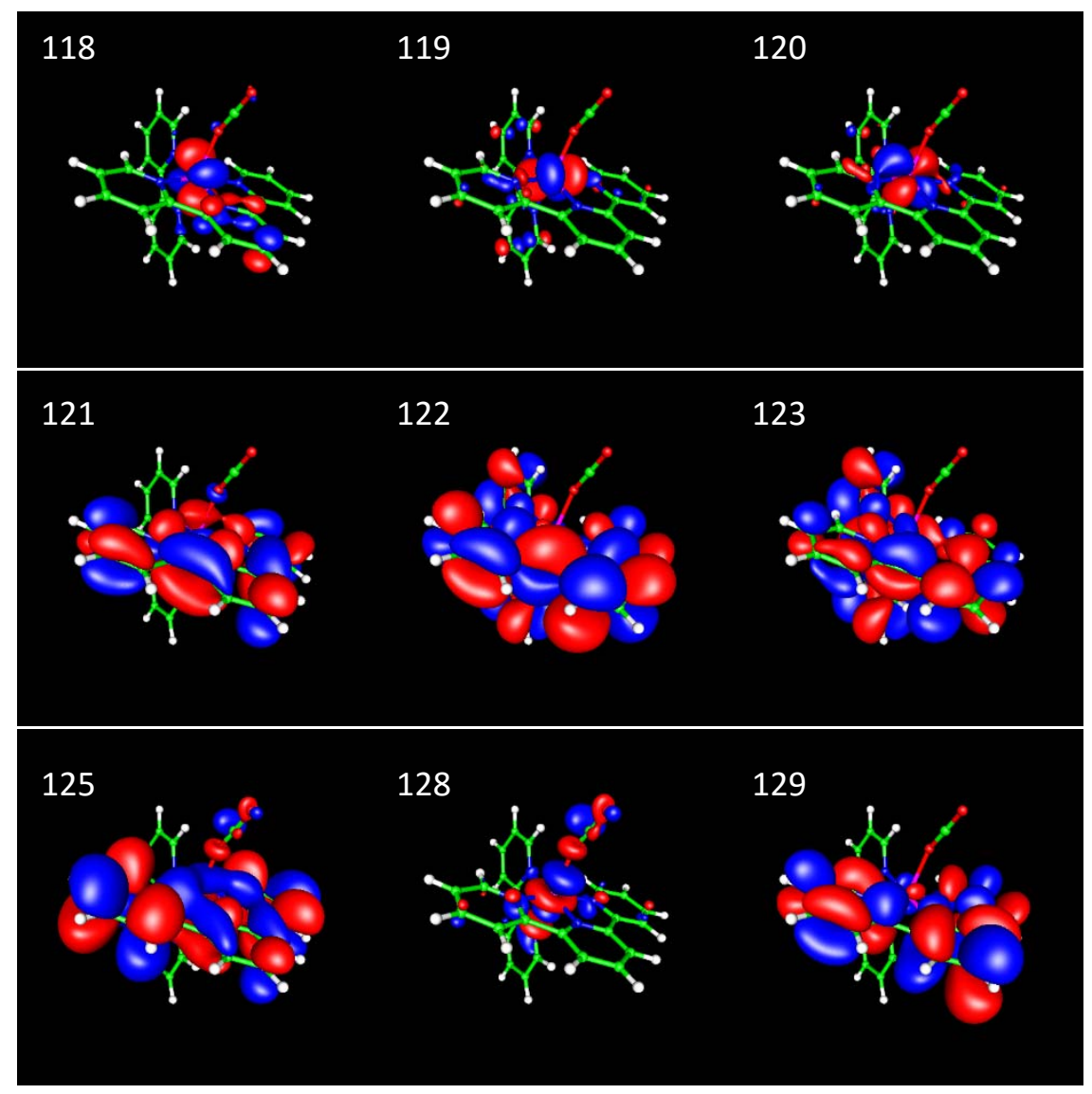


Table S6. Cartesian coordinates of $[\mathbf{R u}-\mathrm{OCO}]^{2+}$ (in Bohr radii).

\begin{tabular}{|c|c|c|}
\hline-0.74266744976876 & -3.88002321560105 & $-0.79137353067778 \mathrm{Ru}$ \\
\hline-1.27165482555484 & -2.22962498258953 & $7.32210939415507 \mathrm{C}$ \\
\hline-3.72120719248453 & -1.30788864799758 & 7.53439875779299 \\
\hline-5.20516076117529 & -1.15897093289976 & 5.38105462230861 \\
\hline-4.22235575439985 & -1.92980598757308 & 3.06396567927009 \\
\hline-0.40216145992286 & -2.967569 & 1075792833 \\
\hline-0.04391176224394 & -2.37969588115757 & $8.94861518646989 \mathrm{I}$ \\
\hline-4.46369463159111 & -0.71480778196972 & $9.34472750674906 \mathrm{I}$ \\
\hline-7.11277644143085 & -0.44583617078474 & $5.51143032602145 \mathrm{I}$ \\
\hline 1.49149307216615 & -3.69492089891692 & $4.72518857567039 \mathrm{H}$ \\
\hline-1.82793381087425 & -2.82970143072622 & $2.87343146311076 \mathrm{I}$ \\
\hline-8.09821450312141 & -0.99538532034901 & 0.44401823357211 \\
\hline-9.278419036 & 3306 & -1.8920340 \\
\hline-7.94116865153369 & -1.834 & -3.98164343059096 \\
\hline-5.48502789570331 & -2.66452155411982 & -3.65759244 \\
\hline-5.62097329181361 & -1.85056211736060 & 7334 \\
\hline-9.10441816482228 & -0.33963641409550 & 2.09367775048305 \\
\hline-11.20380055757910 & -0.32092992262947 & -2.07580368917455 \\
\hline-8.77551386925337 & -1.86256676348792 & -5.84663390985923 \\
\hline-4.39313384601734 & -3.33878349167345 & -5.24263541576967 \\
\hline-4.32672591734922 & -2.68348029061354 & -1.39661591148298 \\
\hline-3.55633956416757 & -11.59135350515930 & 0.74391303113276 \\
\hline-3.13188234394270 & -12.76150159511888 & -1.56124718247356 \\
\hline-2.05302488235118 & -11.37091446707898 & -3.50771835533077 \\
\hline-1.42366616574429 & -8.85209226113284 & -3.12022528002341 \\
\hline-2.89119747765923 & -9.07151713970471 & 1.02156283070016 \\
\hline-4.38933530972152 & -12.59802040035015 & 2.3149064895647 \\
\hline
\end{tabular}




$\begin{array}{llll}-3.62836151688125 & -14.72417627688187 & -1.84777704500855 \mathrm{H} \\ -1.70706492667563 & -12.25037472989266 & -5.31789412569005 \mathrm{H} \\ -3.19198500281052 & -8.09479336882907 & 2.79082949044158 \mathrm{H} \\ -1.85183727439775 & -7.71158687956611 & -0.841966884319548 \mathrm{~N} \\ 0.44176991975815 & -7.97390728426920 & -7.50865782672414 \mathrm{C} \\ 1.55948588870756 & -6.20543160932966 & -9.09816512172929 \mathrm{C} \\ 1.98296367818893 & -3.75067624892414 & -8.26268132602865 \mathrm{C} \\ 1.25905365251473 & -3.09557613668971 & -5.82031409972713 \mathrm{C} \\ -0.25483608325654 & -7.24162124174545 & -5.08037047955718 \mathrm{C} \\ 0.13772573327356 & -9.88447806846111 & -8.15981037969233 \mathrm{H} \\ 2.11438105520855 & -6.74613871720220 & -10.99012606642996 \mathrm{H} \\ 2.87167466324623 & -2.39016868974097 & -9.49808073825050 \mathrm{H} \\ 0.15612560306329 & -4.84141344114534 & -4.32498850949201 \mathrm{~N} \\ 2.64270077563902 & 1.46976411057913 & -5.80080524277460 \mathrm{C} \\ 2.90443658299668 & 3.73812607880192 & -4.50731814562071 \mathrm{C} \\ 2.10059106399628 & 3.87402597498154 & -2.01758025977140 \mathrm{C} \\ 1.05952228943628 & 1.73891877403498 & -0.90924528761095 \mathrm{C} \\ 1.58785871026319 & -0.60829945494055 & -4.59250516468305 \mathrm{C} \\ 3.25957492634992 & 1.32721557446806 & -7.74198576494075 \mathrm{H} \\ 3.72419034196318 & 5.36568241228584 & -5.43448853968190 \mathrm{H} \\ 2.26883250224719 & 5.59800142600964 & -0.93360206788643 \mathrm{H} \\ 0.41240179557110 & 1.77098708666007 & 1.02850127386402 \mathrm{H} \\ 0.79640434913575 & -0.45731795236051 & -2.13783886765267 \mathrm{~N} \\ 5.29035143463029 & -6.03261321480359 & 0.08771399002557 \mathrm{C} \\ 3.28335362776325 & -5.14193038175369 & 0.35337878440520 \mathrm{O} \\ 7.26564570509881 & -6.90064855805156 & -0.14306944469557 \mathrm{O}\end{array}$


Table S7. Lowest energy calculated electronic transition energies and oscillator strengths for $\mathrm{L}=\mathrm{N}_{2}$ (B3LYP functional, unshifted). Only contributions of $10 \%$ and greater are shown.

\begin{tabular}{|c|c|c|c|c|}
\hline Transition & $\begin{array}{c}\mathrm{E}[\mathrm{eV}] / \mathrm{f}\left[10^{-3}\right] \\
\mathrm{B} 3 \mathrm{LYP}\end{array}$ & $\begin{array}{l}\mathrm{E}_{\exp }[\mathrm{eV}] \\
\pm 0.03 \mathrm{eV} \\
\end{array}$ & $\begin{array}{c}\text { orbitals } \\
\text { (see p. S4) }\end{array}$ & character \\
\hline 1 & $2.76 / 0.27$ & & $116 \rightarrow 117(99.1 \%)$ & $\mathrm{d} \rightarrow \operatorname{trpy}$ \\
\hline 2 & $2.95 / 4.6$ & & $115 \rightarrow 117(98.6 \%)$ & $\mathrm{d} \rightarrow \operatorname{trpy}$ \\
\hline 3 & $3.10 / 19.9$ & & $\begin{array}{l}116 \rightarrow 118(65.7 \%) \\
116 \rightarrow 119(22.4 \%)\end{array}$ & d $\rightarrow$ bipy \\
\hline 4 & $3.15 / 2.08$ & & $\begin{array}{l}116 \rightarrow 119(74.5 \%) \\
116 \rightarrow 118(22.3 \%)\end{array}$ & $\mathrm{d} \rightarrow$ trpy \\
\hline 5 & $3.25 / 25.6$ & & $\begin{array}{l}114 \rightarrow 117(65.2 \%) \\
115 \rightarrow 119(27.3 \%)\end{array}$ & $\mathrm{d} \rightarrow \operatorname{trpy}$ \\
\hline 6 & $3.32 / 68.5$ & & $115 \rightarrow 118(81.2 \%)$ & $d \rightarrow$ bipy \\
\hline 7 & $3.39 / 0.24$ & & $\begin{array}{l}114 \rightarrow 118(85.7 \%) \\
114 \rightarrow 119(12.5 \%)\end{array}$ & $d \rightarrow$ bipy \\
\hline 8 & $3.489 / 10.2$ & & $\begin{array}{l}114 \rightarrow 119(64.9 \%) \\
115 \rightarrow 119(12.0 \%)\end{array}$ & $\mathrm{d} \rightarrow$ trpy \\
\hline 9 & $3.492 / 19.7$ & & $\begin{array}{l}116 \rightarrow 119(46.6 \%) \\
114 \rightarrow 117(22.1 \%) \\
114 \rightarrow 119(16.7 \%)\end{array}$ & $\mathrm{d} \rightarrow$ trpy \\
\hline 10 & $3.71 / 0.99$ & & $\begin{array}{l}116 \rightarrow 127(59.9 \%) \\
116 \rightarrow 124(15.0 \%) \\
116 \rightarrow 128(13.1 \%)\end{array}$ & $\mathrm{d} \rightarrow \mathrm{N}_{2}$ \\
\hline
\end{tabular}


Figure S5. Calculated orbitals contributing to transitions for $\mathrm{L}=\mathrm{N}_{2}$. Orbital numbers are given as mentioned in Table S7.

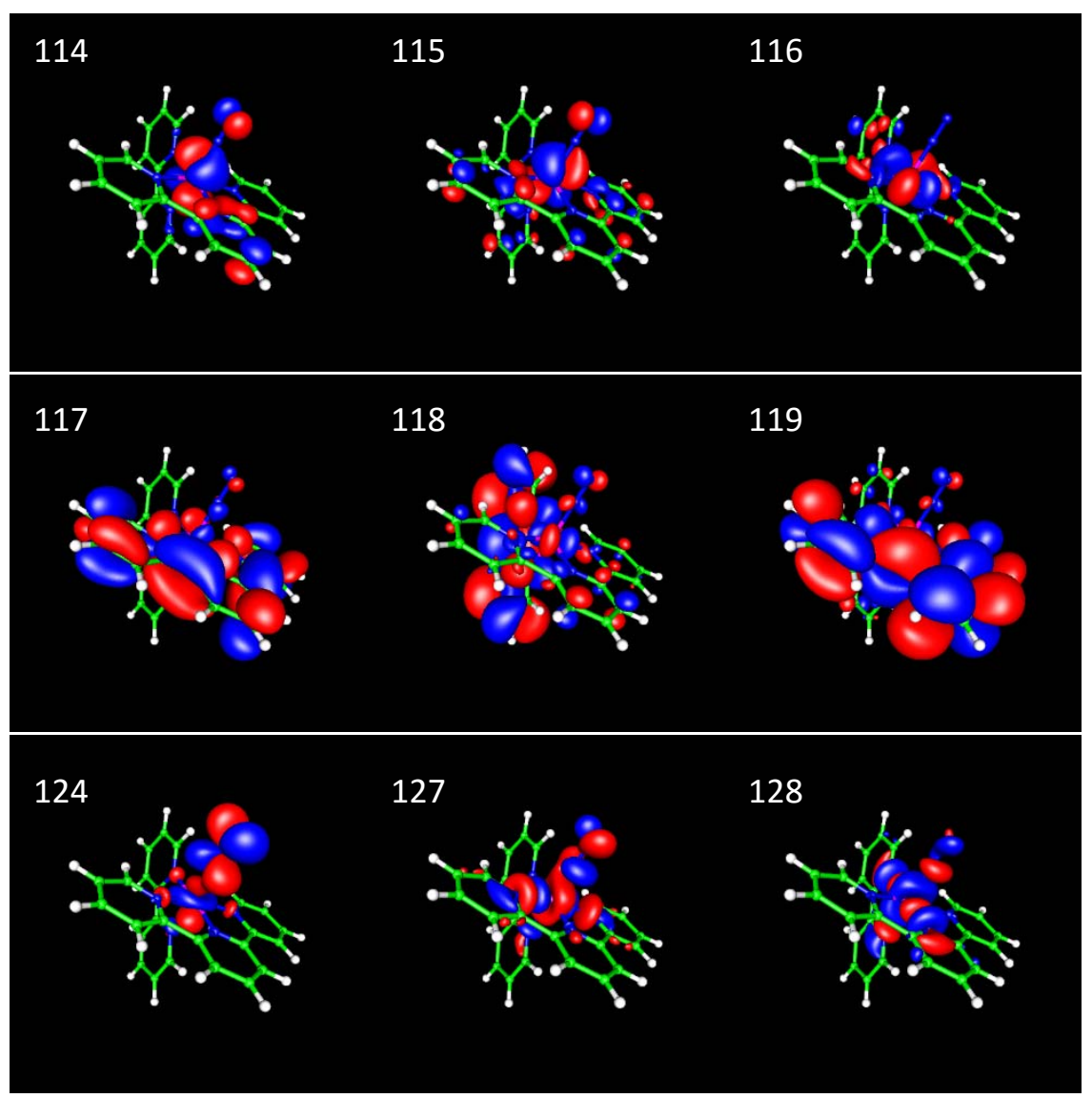


Table S8. Cartesian coordinates of $\left[\mathbf{R u}-\mathrm{N}_{2}\right]^{2+}$ (in Bohr radii).

\begin{tabular}{|c|c|c|}
\hline-0.62183235481201 & -4.07197880692217 & $-0.83278599512107 \mathrm{R}$ \\
\hline-1.21153812643367 & -2.43206021717827 & $7.30759989586031 \mathrm{C}$ \\
\hline-3.64596629466877 & -1.47527072426499 & 7.51052191534002 \\
\hline-5.11133622634736 & -1.29256794388958 & 5.34711535062101 \\
\hline-4.13059941319450 & -2.06394024329056 & 3.02930647205915 \\
\hline-0.340 & 99889877 & $7317 \mathrm{C}$ \\
\hline 0.00708231132598 & -2.61198238725848 & $861 \mathrm{H}$ \\
\hline-4.39286805501384 & -0.88128807704715 & $9.31883717970571 \mathrm{H}$ \\
\hline-7.00787967588540 & -0.55090186320116 & $5.47181040561139 \mathrm{H}$ \\
\hline 1.544 & -3.92 & $5768880 \mathrm{H}$ \\
\hline-1.74793680955949 & -3.00022934416906 & $2.84747956858238 \mathrm{~N}$ \\
\hline-8.00791385300529 & -1.02550854300802 & $0.45180978268652 \mathrm{C}$ \\
\hline 8751 & -0.9630 & $245 \mathrm{C}$ \\
\hline-7.91123364694089 & -1.81686209520313 & -3.9880097 \\
\hline-5.46915847635320 & -2.69996347858457 & $-3.68945557997591 \mathrm{C}$ \\
\hline-5.54545506303053 & -1.93488617713165 & $0.63867065198573 \mathrm{C}$ \\
\hline-8.989865787 & -0.366102 & $2.11388199603877 \mathrm{H}$ \\
\hline 11.12119769046538 & -0.25927947561799 & -2.031031 \\
\hline-8.76477148231173 & -1.80530797997674 & $-5.84430756625903 \mathrm{H}$ \\
\hline-4.40619807359074 & -3.37822359137766 & $-5.29413881904020 \mathrm{H}$ \\
\hline-4.29431370227627 & -2.76772351456188 & $-1.44321832182411 \mathrm{~N}$ \\
\hline-3.63370606851986 & -11.73654744427037 & 0.63497559944028 \\
\hline-3.30684111574098 & -12.86997267880518 & -1.70340394638011 \\
\hline-2.25400008397362 & -11.46900752136415 & -3.65668824315152 \\
\hline-1.55099870733646 & -8.97451568126539 & -3.24193542926909 \\
\hline-2.89847682998766 & -9.23877279241710 & 0.93886977019481 \\
\hline .43850066729287 & -12.75496889682852 & 2.2129801409234 \\
\hline
\end{tabular}




$\begin{array}{llll}-3.85508040814439 & -14.81578090017718 & -2.01013450282723 \mathrm{H} \\ -1.98057101115046 & -12.32402049665021 & -5.49061759068845 \mathrm{H} \\ -3.11491282924324 & -8.29034003685321 & 2.73553273212611 \mathrm{H} \\ -1.88598381765038 & -7.87361420883015 & -0.93185887446081 \mathrm{~N} \\ 0.15760778566336 & -8.05226509839726 & -7.68382811706964 \mathrm{C} \\ 1.25463833243355 & -6.28000365605011 & -9.28260365405125 \mathrm{C} \\ 1.77666533757601 & -3.85370475469408 & -8.42106423400790 \mathrm{C} \\ 1.18619325362972 & -3.23494901623478 & -5.93349889863863 \mathrm{C} \\ -0.40385059777636 & -7.35619899904058 & -5.21003637337362 \mathrm{C} \\ -0.23560334998141 & -9.93636860117353 & -8.36325078346785 \mathrm{H} \\ 1.70974110904776 & -6.79422660754394 & -11.20840492954842 \mathrm{H} \\ 2.63592289410428 & -2.48809563515582 & -9.67127648526129 \mathrm{H} \\ 0.12297286903145 & -4.99134751144699 & -4.42839057852635 \mathrm{~N} \\ 2.67781133005911 & 1.29562515126359 & -5.89725828286008 \mathrm{C} \\ 3.03136270918560 & 3.54050929618896 & -4.58469487709621 \mathrm{C} \\ 2.30661901573237 & 3.66529648917666 & -2.07083669911432 \mathrm{C} \\ 1.25167423146176 & 1.53986372767028 & -0.95595864852183 \mathrm{C} \\ 1.61323953825927 & -0.77301889813578 & -4.68203111996120 \mathrm{C} \\ 3.23655604652830 & 1.16291699029769 & -7.85643683168419 \mathrm{H} \\ 3.86494784297488 & 5.15859008629996 & -5.51619396432434 \mathrm{H} \\ 2.55167917089916 & 5.36994819899085 & -0.97121313463810 \mathrm{H} \\ 0.67055027957885 & 1.55875090329066 & 1.00269141003419 \mathrm{H} \\ 0.90217833805358 & -0.62964697397829 & -2.20498612303463 \mathrm{~N} \\ 2.80578903147978 & -5.21589353374673 & 0.13056454883807 \mathrm{~N} \\ 4.71574723020939 & -5.85335584791364 & 0.66040295694251 \mathrm{~N}\end{array}$


Table S9. Lowest energy calculated electronic transition energies and oscillator strengths for $\mathbf{R u}^{2+}$ (B3LYP functional, unshifted). Only contributions of $10 \%$ and greater are shown.

\begin{tabular}{|c|c|c|c|c|}
\hline Transition & $\begin{array}{c}\mathrm{E}[\mathrm{eV}] / \mathrm{f}\left[10^{-3}\right] \\
\mathrm{B} 3 \mathrm{LYP}\end{array}$ & $\begin{array}{c}\mathrm{E}_{\exp }[\mathrm{eV}] \\
\pm 0.03 \mathrm{eV}\end{array}$ & $\begin{array}{c}\text { orbitals } \\
\text { (see p. S4) }\end{array}$ & character \\
\hline 1 & $1.73 / 9.62$ & & $\begin{array}{l}109 \rightarrow 110(71.1 \%) \\
109 \rightarrow 113(13.0 \%)\end{array}$ & $\mathrm{d} \rightarrow \operatorname{trpy}$ \\
\hline 2 & $1.84 / 1.08$ & & $\begin{array}{l}108 \rightarrow 110(10.7 \%) \\
108 \rightarrow 110(69.1 \%) \\
108 \rightarrow 113(14.5 \%) \\
109 \rightarrow 110(14.2 \%)\end{array}$ & \\
\hline 3 & $2.06 / 10.2$ & & $\begin{array}{l}107 \rightarrow 110(70.3 \%) \\
107 \rightarrow 113(25.5 \%)\end{array}$ & \\
\hline 4 & $2.80 / 2.21$ & & $\begin{array}{l}109 \rightarrow 111(80.4 \%) \\
109 \rightarrow 112(15.7 \%)\end{array}$ & $\mathrm{d} \rightarrow$ bipy/trpy \\
\hline 5 & $2.86 / 0.44$ & & $\begin{array}{l}109 \rightarrow 113(72.4 \%) \\
109 \rightarrow 110(12.4 \%)\end{array}$ & $\mathrm{d} \rightarrow \operatorname{trpy}$ \\
\hline 6 & $2.91 / 1.1$ & & $\begin{array}{l}108 \rightarrow 113(72.1 \%) \\
108 \rightarrow 110(14.8 \%)\end{array}$ & $\mathrm{d} \rightarrow \operatorname{trpy}$ \\
\hline 7 & $2.97 / 2.77$ & & $\begin{array}{l}109 \rightarrow 112(75.2 \%) \\
109 \rightarrow 111(15.0 \%)\end{array}$ & $\mathrm{d} \rightarrow$ bipy/trpy \\
\hline 8 & $3.07 / 85.6$ & & $\begin{array}{l}108 \rightarrow 111(81.5 \%) \\
108 \rightarrow 112(11.7 \%)\end{array}$ & \\
\hline 9 & $3.105 / 32.2$ & & $\begin{array}{l}108 \rightarrow 112(54.6 \%) \\
107 \rightarrow 113(25.0 \%)\end{array}$ & \\
\hline 10 & $3.114 / 0.04$ & & $\begin{array}{l}107 \rightarrow 111(84.3 \%) \\
107 \rightarrow 112(10.9 \%)\end{array}$ & \\
\hline 11 & $3.27 / 1.2$ & & $\begin{array}{l}107 \rightarrow 112(76.7 \%) \\
106 \rightarrow 110(10.9 \%) \\
107 \rightarrow 111(10.7 \%)\end{array}$ & \\
\hline 12 & $3.35 / 15.6$ & & $\begin{array}{l}107 \rightarrow 113(40.0 \%) \\
108 \rightarrow 112(23.0 \%) \\
107 \rightarrow 110(17.0 \%)\end{array}$ & \\
\hline 13 & $3.49 / 132.7$ & & $106 \rightarrow 110(81.9 \%)$ & \\
\hline 14 & $3.60 / 7.45$ & & $105 \rightarrow 110(97.5 \%)$ & \\
\hline 15 & $3.74 / 2.48$ & & $109 \rightarrow 114(93.3 \%)$ & \\
\hline
\end{tabular}


Figure S6. Calculated orbitals contributing to transitions for $\mathrm{L}=\mathrm{N}_{2}$. Orbital numbers are given as mentioned in Table S9.
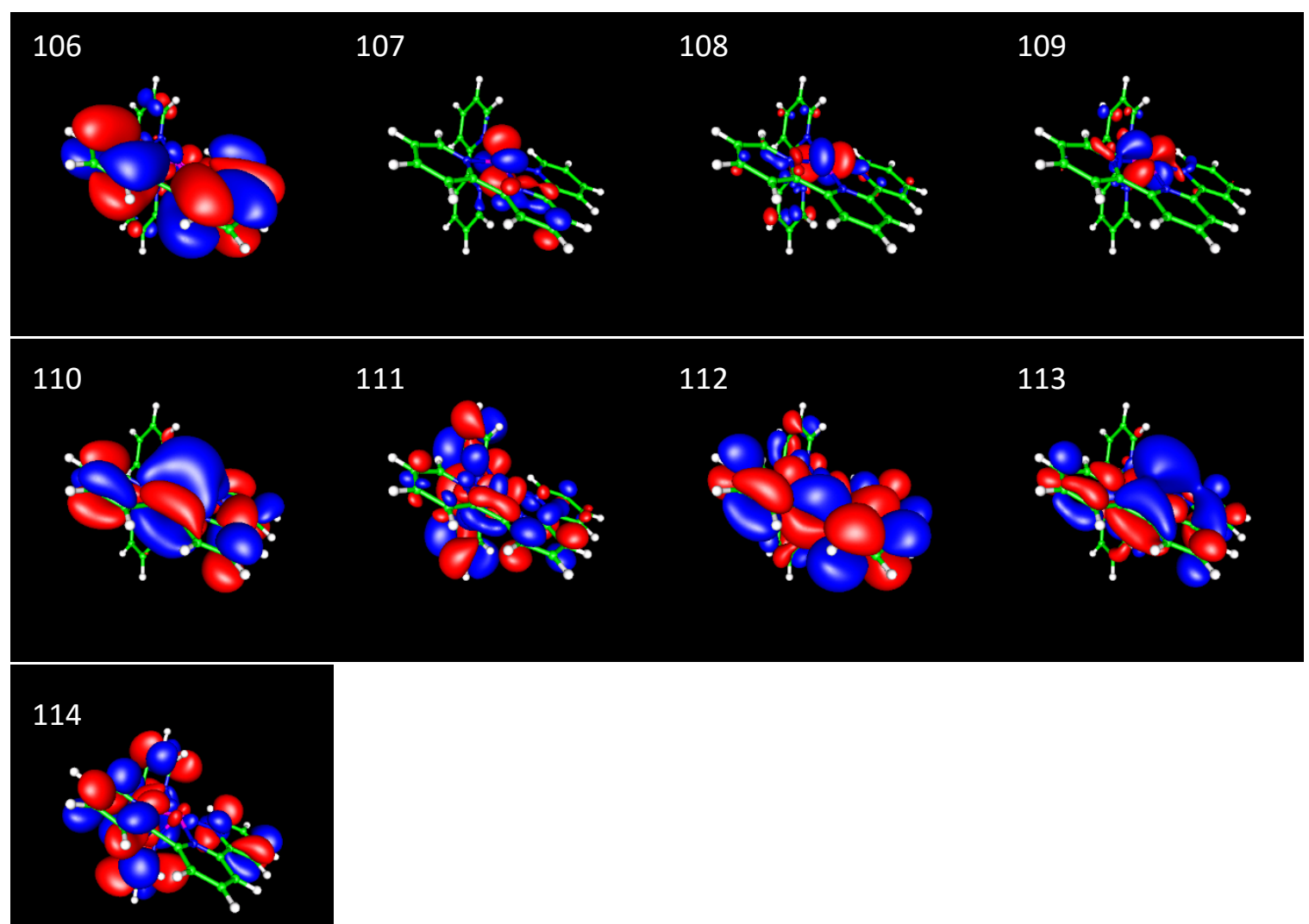
Table S10. Cartesian coordinates of $\mathbf{R u}^{2+}$ (in Bohr radii).

\begin{tabular}{|c|c|c|}
\hline-0.93230289535152 & -3.90504272656346 & -0.96790877835039 \\
\hline-1.04577679453402 & -2.45357262439691 & 7.16688378832307 \\
\hline-3.45538851711131 & -1.46833169375499 & 7.51831046054781 \\
\hline-5.03819994059482 & -1.21825047970586 & 5.445124 \\
\hline-4.18 & -1.95 & 518921363 \\
\hline-0.309089713 & -3.15174620825289 & 4.75 \\
\hline 0.25127831140173 & -2.68167402803020 & 8.72 \\
\hline-4.09181746593699 & -0.90346478856401 & \\
\hline & -0.4 & \\
\hline 1.55 & -3.9 & \\
\hline-1.83213554963756 & -2.91495594931444 & 2.7416531 \\
\hline-8.12854289758138 & -0.86773255660406 & 0.5 \\
\hline-9.37 & -0.7 & -1 . \\
\hline-8.13018198366255 & -1.631938576 & -3.8 \\
\hline-5.68788354371253 & -2.53167375607722 & -3.6 \\
\hline-5.66934018022202 & -1.7892 & \\
\hline-9.06 & -0.2171 & \\
\hline-11.29024490673315 & -0.06893636708358 & \\
\hline-9.02343035536709 & -1.60482104275015 & \\
\hline-4.65801309345598 & -3.20665180526 & -5.2 \\
\hline-4.46696000956950 & -2.61767290930126 & -1.39268663685775 \\
\hline-3.73901850734446 & -11.61086669521778 & \\
\hline-3.29497086945966 & -12.79285921774863 & -1.7066806550233 \\
\hline-2.20688055791263 & -11.41094171406364 & -3.6560819990042 \\
\hline-1.58879349635309 & -8.88961397340942 & -3.2809629931088 \\
\hline-3.08822197940921 & -9.08572322503805 & 0.8525908515657 \\
\hline
\end{tabular}




\begin{tabular}{rrrrr}
-4.57636443370310 & -12.61127923220470 & $2.16036160535171 \mathrm{H}$ \\
-3.78112750112478 & -14.75949656493148 & $-1.98392838065187 \mathrm{H}$ \\
-1.84232283363943 & -12.30240298027843 & $-5.45661714812907 \mathrm{H}$ \\
-3.40598248412698 & -8.09891929918945 & $2.61321335704908 \mathrm{H}$ \\
-2.04173392344523 & -7.73341725842066 & $-1.01356363166069 \mathrm{~N}$ \\
0.40262151302245 & -8.06237092881420 & $-7.62289348215732 \mathrm{C}$ \\
1.59053265571809 & -6.31745036019155 & $-9.18786611435708 \mathrm{C}$ \\
2.01739198474493 & -3.86041782337447 & $-8.35987631484810 \mathrm{C}$ \\
1.20749892771370 & -3.17375483031382 & $-5.95506684572581 \mathrm{C}$ \\
-0.37802681275341 & -7.29720800469447 & $-5.23213271141505 \mathrm{C}$ \\
0.11878729496639 & -9.98022462393808 & $-8.26140144831933 \mathrm{H}$ \\
\hline 2.21109663089563 & -6.88327001354793 & $-11.05197877013946 \mathrm{H}$ \\
\hline 2.98171449475164 & -2.52796531733007 & $-9.56873025823841 \mathrm{H}$ \\
\hline 0.01040667647515 & -4.88736836328045 & $-4.49525719520649 \mathrm{~N}$ \\
\hline 2.70517181799177 & 1.35448333540959 & $-5.89535345591106 \mathrm{C}$ \\
\hline 3.01569250506847 & 3.60802678485608 & $-4.58397891958945 \mathrm{C}$ \\
\hline 3.17242116406146 & 3.75855615315319 & $-2.10900273627085 \mathrm{C}$ \\
\hline 1.04029348716867 & 1.65373587056266 & $-1.03217382248024 \mathrm{C}$ \\
\hline .56378404078520 & -0.69317366684055 & $-4.71872529403023 \mathrm{C}$ \\
\hline .35589761698273 & 1.20095036105355 & $-7.82453499787380 \mathrm{H}$ \\
\hline
\end{tabular}

